\title{
Negative Pressure Regimes in Ionic Liquids: Structure and Interactions in Stretched Liquids as Probed by NMR
}

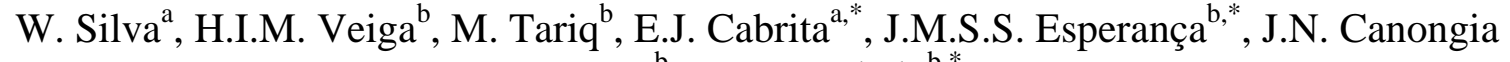 \\ Lopes $^{\mathrm{b}}$, L.P.N. Rebelo ${ }^{\mathrm{b}, *}$ \\ ${ }^{a}$ UCIBIO, REQUIMTE, Departamento de Química, Faculdade de Ciências e Tecnologia, \\ Universidade Nova de Lisboa, Caparica, 2829-516, Portugal \\ ${ }^{\mathrm{b}}$ LAQV, REQUIMTE, Departamento de Química, Faculdade de Ciências e Tecnologia, \\ Universidade Nova de Lisboa, Caparica, 2829-516, Portugal \\ * luis.rebelo@ @ct.unl.pt; ejc@ @fct.unl.pt; jmesp@fct.unl.pt
}

In this work, a broad pallete of macroscopically sized samples of ionic liquids was used, in which tensions of about -100 MPa were obtained in preliminary testing methodologies before cavitation would occur. Preliminary NMR studies have been performed on ionic liquids under homogeneous tension. Upon entering into these metastable regimes of negative pressure, it is possible to observe a sharp increase in the anion and cation self-diffusion coefficients as compared to the equilibrium saturated liquid conditions at identical temperatures. [L02 - Molten Salts and Ionic Liquids 21, Structure and Properties I, Tuesday, October $2^{\text {nd }}, 10: 40$, \#1832-AiMES2018, Universal 9 - Abstract Oral \#116064 ]

\section{Introduction}

Condensed matter - solids or liquids, but not gases - might be deeply, homogeneously stretched to absolute negative pressures (metastable regimes of isotropic tension). For instance, we were able to stretch macroscopically-sized liquid solutions down to $\mathrm{p} \sim-35$ $\mathrm{MPa},(1,2)$ a value which still constitutes a world-record for very large volume liquid samples. Very recently, we successfully obtained a consistent set of tensions of about $100 \mathrm{MPa}$ in a variety of commonly used ionic liquids (ILs) in Pyrex glass capillaries of internal volume of $\sim 0.05 \mathrm{~cm}^{3}$. (3) Up to now, the absolute record for liquids is kept for neat liquid water within much smaller volumes - microscopic porous crevices of naturally occurring rocks, $p \sim-150 \mathrm{MPa}$, a value which is believed to be not far from the limit of intrinsic, ultimate tensile strength of water.(4) In this work, we describe the change on the fluid molecular dynamics (transport response functions) of liquid salts upon applied homogeneous tension using pulsed field gradient NMR spectroscopy.

\section{Experimental}

There are several strategies to force a liquid entering into negative pressure regimes. The most common one is the well-known Berthelot method (Figure 1).(5) Negative pressures can be either directly measured - via a highly elaborated Bourdon-Berthelot combination methodology (Figure 2),(5-7) or safely, though approximately, estimated through the liquid's isochoric thermal-pressure coefficient, $\gamma_{\mathrm{v}}=\alpha_{\mathrm{p}} / \kappa_{\mathrm{T}}$. 


$$
\begin{aligned}
& \gamma_{v}=\left(\frac{\partial p}{\partial T}\right)_{v}=-(\partial V / \partial T)_{p} /(\partial V / \partial p)_{T}=\frac{\alpha_{p}}{K_{T}} \\
& P=P_{\text {fill }}+\int_{T_{\text {fil }}}^{T} \gamma_{v} d T \approx P_{\text {fill }}+\gamma_{v}\left(T-T_{\text {fill }}\right)
\end{aligned}
$$

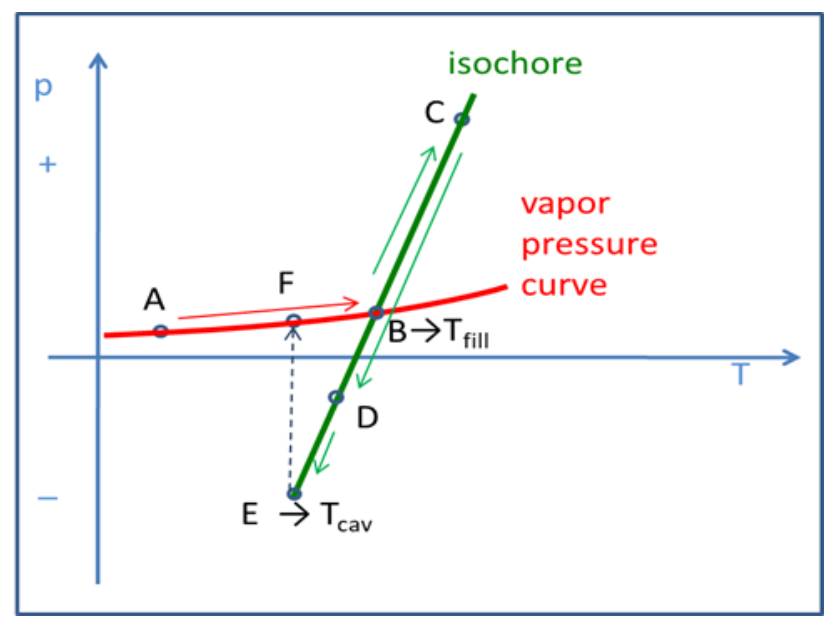

Figure 1. The Berthelot cycle: Liquid inside its container (typically a glass capillary) at its L-V equilibrium (A) - for ILs this vapour pressure line is, nominally, a zero pressure isobar. Upon temperature increase, by isobaric thermal expansion, the liquid fills in the entire internal volume at $T_{\text {fill }}(\mathrm{B})$. Continuing increasing temperature, forces the liquid to evolve along an isochore (constant volume) at concomitantly higher, positive pressures (C) - the so-called, pre-pressurization stage. Reverting the temperature-change direction, and assuming the liquid adheres extremely well to the glass capillary walls, at point (D) the liquid is experiencing an isotropic, bulk tension (absolute negative pressure metastable state). Eventually, at $T_{\text {cav }}$ (cavitation temperature) it will collapse at point (E), relaxing back to its stable condition located along its vapour pressure curve at $(\mathrm{F})$.
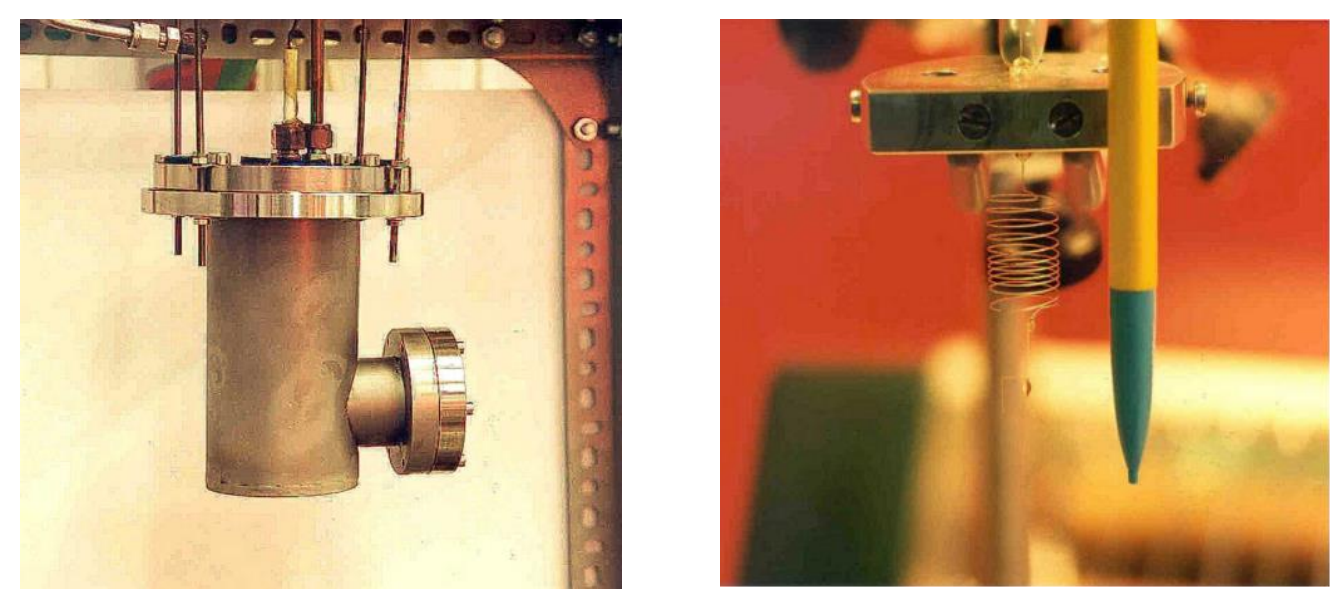

Figure 2. Apparatus for direct measurements of negative pressures: (left) external apparatus' body, and (right) detail of the helix glass capillary with a small glass mirror hanging on its bottom end. Positive or negative pressures will make the coil expand or contract, provoking a rotation movement of the mirror either in the clockwise or anticlockwise direction. 
Self-diffusion measurements have been performed by pulsed field gradient NMR using a stimulated echo and bipolar gradient pulses - in a Bruker Avance III $400 \mathrm{MHz}$ instrument, equipped with a BBO probe, and using a gradient $=50.0 \mathrm{G} / \mathrm{cm}, \Delta=300-$ $700 \mathrm{~ms}$, and $\delta=4-6 \mathrm{~ms}$. Temperatures were both certified by internal NMR calibration as well as by thermodynamic melting point observation of reference chemical compounds.

Pyrex glass capillaries, sealed at both ends, of internal volume of $\sim 0.05 \mathrm{~cm}^{3}$, were placed inside normal NMR tubes and data were taken for each temperature for about an initial equilibration temperature period of $30 \mathrm{~min}$. A Berthelot-type of cycle (Fig. 1) was applied and pressures were estimated through equations [1-2].

\section{Discussion and Conclusions}

In this work, a broad pallete of macroscopically sized samples of ionic liquids was used, in which tensions of about $-100 \mathrm{MPa}$ were obtained in preliminary testing methodologies before cavitation would occur. This value constitutes a new record for large samples $(0.05 \mathrm{ml})$. The underlying success for achieving such great absolute negative pressures in ionic liquids as compared to other liquids (including water) seems to be the very best combination of (i) their negligible vapour pressure, (ii) their generally high viscosity, (iii) their relatively small surface tension, (iv) their good adhesion (wettability) to the container's walls (in this case Pyrex glass), and (v) their easy invasion into their stable (but difficult to be kinetically achieved) crystalline territory.

The collapse of a metastable liquid under tension that relaxes to its stable L-V condition at a given temperature is a phenomenon that, at a practical level, has little to do to its intrinsic homogeneous limit of mechanical instability (the spinodal line). For water, this ultimate limit is intimately related to the merge of its temperature of maximum density (TMD) $p$ - $T$ locus with the L-V spinodal, which may be either re-tracking back at lower temperatures to the supercooled liquid regime or might be facing an "inflexion point plateau". That limit seems to be located at around $-150 \mathrm{MPa}\left(\right.$ for $\mathrm{H}_{2} \mathrm{O}$ or $\mathrm{D}_{2} \mathrm{O}$ ), at a mild temperature, not far from room-temperature.

Under this practical viewpoint, the liquid suffers cavitation well before (at less extreme conditions) reaching its limit of intrinsic (bulk) tension and concomitant collapse. Possible reasons include either any external perturbation such as the always-present mechanical vibrations, or natural cosmic rays bombardment, etc. However, more importantly, most often, a weak adhesion to the container's walls, the presence of microand nano-gas phase bubbles in its milieu, its interfacial tension which leaves at the crevices of the container's walls "empty spaces", and the relatively high liquids' dynamics (low viscosity, high self-diffusion coefficient) which eases up their vaporization (cavitation) process, are the major key-factors responsible for unsuccessful liquid stretches.

Generally, aprotic ionic liquids are viscous fluids (100 - 1000 times more "immobile" than water), presenting relatively low surface tensions,(8) and, not the least, confined to a vapour pressure near to vacuum conditions (almost no gas bubbles are present in the bulk).(9,10) Moreover, most of them easily supercool, well deeper into their stable crystalline regimes, and much more deeply than most fluids can do. All these characteristics, allied to the fact that they do not present the limitation of the existence of any TMD locus, and to that one that is their incredibly high cohesive energy due to the 
presence of strong electrostatic, attractive forces, makes them the ideal fluids to studying the ultimate limits of liquids' stability (instability) amongst any sort of chemical matter known up to present.

Metastability is a relative concept. One must specify under which thermodynamic constraints it is being defined/described.(11) Herein, we are comparing the ionic liquids behaviour under deep absolute negative pressure with that at identical temperature at stable L-V equilibrium conditions (nominal, isobaric pressure of $p=0 \mathrm{MPa}$, same total volume, smaller liquid molar volume). Please bear in mind, that a stretched liquid is expanded as compared to its stable equilibrium state.

For the first time, preliminary NMR studies have been performed on ionic liquids under homogeneous tension. Due to potential hazard issues related to the protection/safety of the NMR instrumentation, negative pressures beyond the value of $20 \mathrm{MPa}$ were avoided (not always in a successful fashion).

Upon entering into metastable regimes of negative pressure, it was possible to observe a sharp increase in the anion and cation self-diffusion coefficients as compared to the equilibrium saturated liquid conditions at identical temperatures.

The thermodynamic Berthelot cycle illustrated in Figure 1 has been used to both promote pre-pressurisation stages as well as homogeneous tensions to negative pressure regimes. Negative pressures have been safely and accurately estimated (equations [1] and [2]) by experimental determination of thermal-pressure coefficients of each liquid at all $T_{\text {fill }}$ relevant-temperatures of the corresponding isochore (invariant, liquid molar volume), inherent to each working capillary, to each IL, and to each temperature.

In the material that follows, one example, for a prototype $\mathrm{IL},\left[\mathrm{C}_{2} \mathrm{C}_{1} \mathrm{im}\right]\left[\mathrm{C}_{2} \mathrm{SO}_{4}\right]$, is provided.

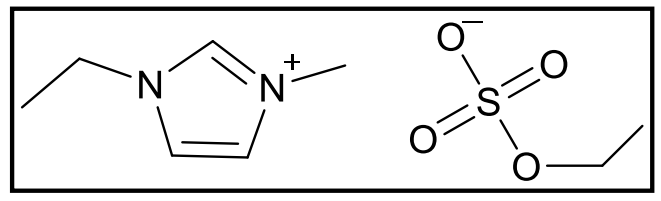

Figure 3. Structural chemical formula of $\left[\mathrm{C}_{2} \mathrm{C}_{1} \mathrm{im}\right]\left[\mathrm{C}_{2} \mathrm{SO}_{4}\right]$.

In one of its glass capillary enclosures, its $T_{\text {fill }}$ was equal to $307.15 \mathrm{~K}$, to which corresponds a thermal-pressure coefficient, $\gamma_{\mathrm{v}}=16.4 \mathrm{bar} / \mathrm{K}$. The ions' absolute selfdiffusion coefficients, $D\left(\mathrm{~m}^{2} \cdot \mathrm{s}^{-1}\right)$, are presented in Figure 4 for the isobaric, nominal zero (vapour) pressure under L-V equilibrium conditions.

Figure 5 shows, for the cation's example, how its self-diffusion markedly decreases upon applied positive pressure (temperatures greater than $T_{\text {fill }}$ ), and how it increases under homogeneous tension in negative pressure regimes $\left(T<T_{\text {fill }}\right)-$ results presented as the ratio $D_{\text {isochore }} / D_{\text {isobar }}$, that is to say at positive or negative pressure as compared to null vapour pressure conditions.

Note that for temperatures below $25.5^{\circ} \mathrm{C}$ ( $p \sim-150$ bar), the liquid salt has already collapsed (cavitated to the L-V condition below this $T_{\text {cav }}$ ), and, therefore, the ratio in $D$ 's was restored to 1.0 .

Extrapolations of $D$ versus $T$ along the isochores using VFT-type of equations which are adequate for glass-forming liquids, have shown a predicted glass transition, $T_{\mathrm{g}}$, for this IL of about $157 \mathrm{~K}$ at a negative pressure of $p=-2500$ bar, a most probably realistic 
value for a highly cohesive liquid without any thermodynamic TMD restriction. The $T_{\mathrm{g}}-p$ slope seems to be similar (though slightly lower) to that of its melting, $T_{\mathrm{m}}-p$.

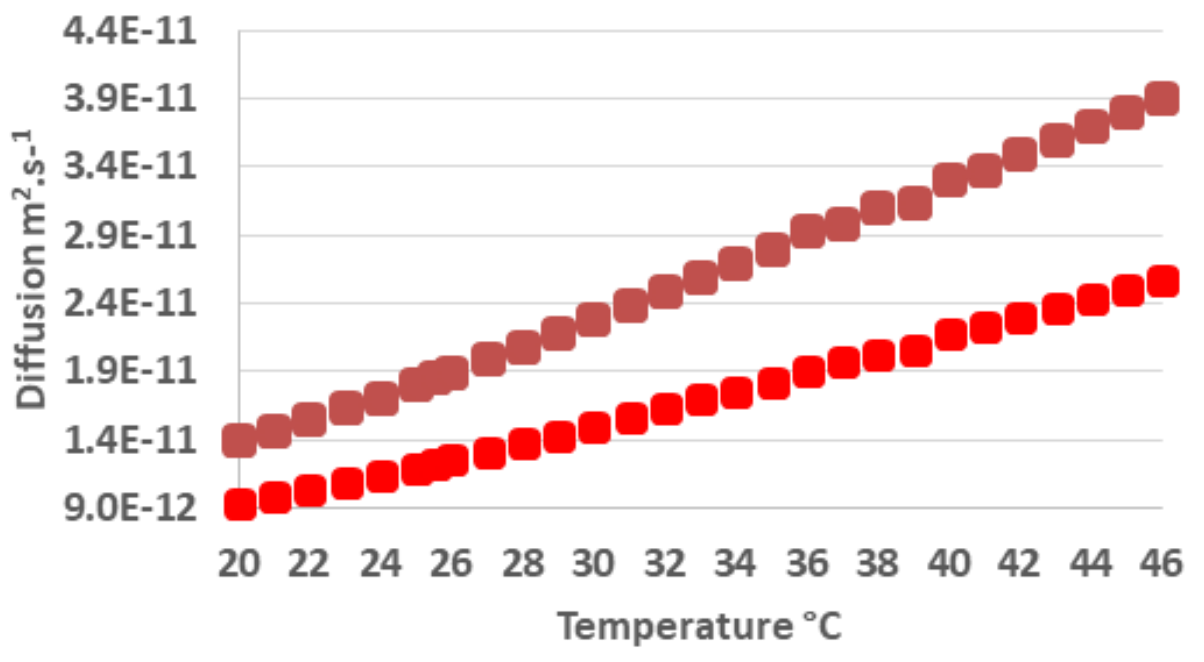

Anion isobaric Cation Isobaric

Figure 4. Self-diffusion coefficients of cation and anion, respectively, $\left[\mathrm{C}_{2} \mathrm{C}_{1} \mathrm{im}\right]^{+}$and $\left[\mathrm{C}_{2} \mathrm{SO}_{4}\right]^{-}$.

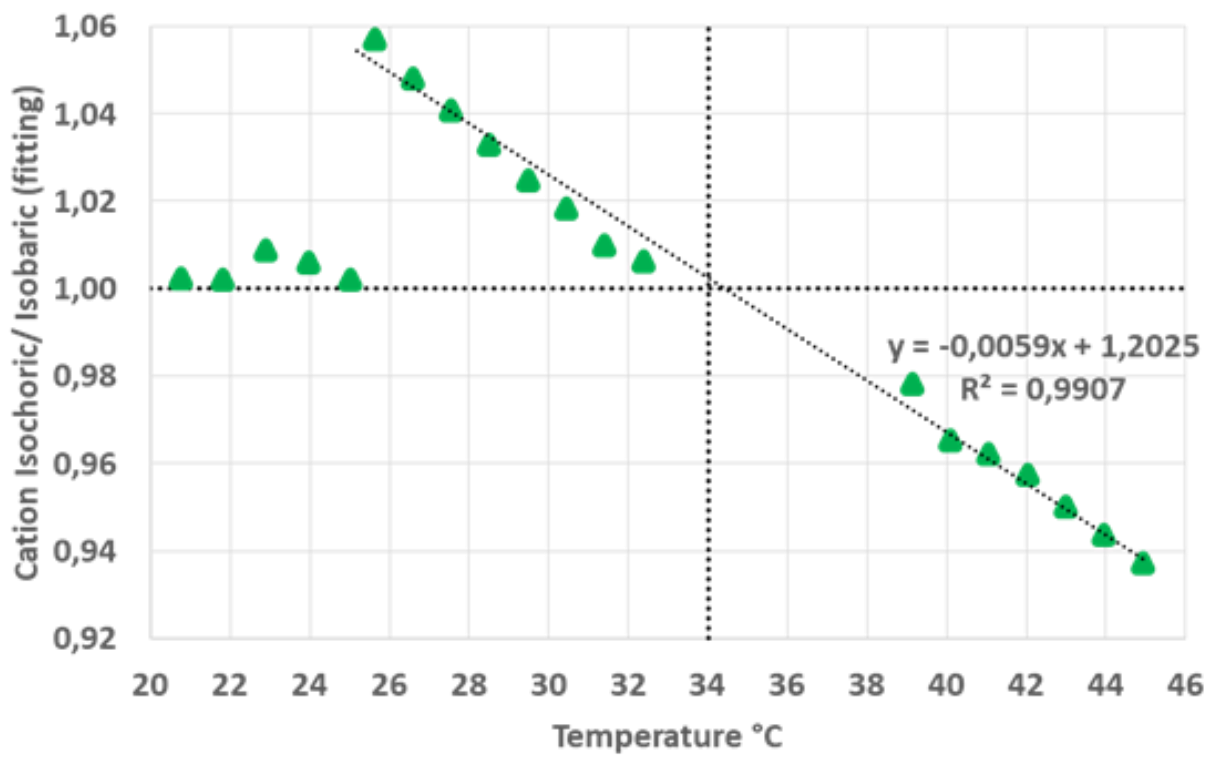

$\triangle$ Diffusion Cation Isochoric/Isobaric (fitting)

Figure 5. Relative cation's self-diffusion coefficients versus temperature in positive, negative, and null pressure regimes. 
MD simulations have been performed to gain insights into the ILs structure and interactions under these deep metastable conditions. The point herein to be made is that, along an isochore (invariant liquid molar volume), as that represented by the C-B-D-E projected $p-T$ line of Figure 1, both the charge ordering peaks (COP) and the contact peaks $(\mathrm{CP})$ of the X-ray structure function, $S(\mathrm{q})$, should also be quasi-invariant. Therefore, one only ought to compare $q\left(\AA^{-1}\right)$ peak positions at two single temperatures (irrespective of pressure): those at $T_{\text {fill }}$ (and $p=0$ ) with those at the cavitation one ( $\left.T_{\text {cav }}\right)$ and $p=0$, because the former represents the structure of the liquid at deep negative pressure at $T_{\text {cav }}$. Therefore, basically, at temperatures (for the L-V isobaric $p=0$ nominal line) of points B and $\mathrm{E}$ (or F) in Figure 1.

Consistent with direct thermodynamic $p-V$ - $T$ data (using the experimental liquid's isothermal compression coefficient, $\left.K_{\mathrm{T}}\right)$, if one takes the typical $q\left(\AA^{-1}\right)$ versus $\mathrm{T}$ dependence of archetypal ILs,(12) it is possible to estimate that these liquid salts expand, isothermally, and under isotropic tension, some mild $0.6-0.7 \%(\Delta V / V \%)$ per each $\Delta p=$ -100 bar of applied negative pressure. Ten times more pronounced (about $5 \%$ per each $\Delta p$ $=-100$ bar) is the concomitant increase in the self-diffusion coefficients of both the anion and the cation of the salt (see Fig. 5).

We anticipate that a similar marked effect might be also observed in the mobility of the ions in ionic liquids aqueous solutions, a fact which is potentially relevant for phenomena dependent on the ions' mobility.

\section{Acknowledgments}

The authors thank Fundação para a Ciência e Tecnologia, FCT/MCTES (Portugal) for financial support through a FCT Investigator contract (IF/00355/2012 - J. M. S. S. E), and through projects UID/QUI/50006/2013 and UID/Multi/04378/2013. This work has been also supported by CNPq - Brazil through grant 201863/2014 - 6 (W. Silva). The NMR spectrometers are part of the National NMR Network (PTNMR) and are supported by Infrastructure Project $\mathrm{N}^{\circ} 022161$ (co - financed by FEDER through COMPETE 2020, POCI, and PORL and FCT through PIDDAC).

\section{References}

1. Z.P. Visak, L.P.N. Rebelo and J. Szydlowski, J. Phys. Chem. B, 107, 9837-9846 (2003).

2. Z.P. Visak, L.P.N. Rebelo and J. Szydlowski, J. Chem Educ., 79, 869-873 (2002).

3. Yet unpublished results; data presented at recent international conferences.

4. Q. Zheng, D.J. Durben, G.H. Wolf and C.A. Angell, Science, 254, 829-832 (1991).

5. A. Imre, K. Martinás and L.P.N. Rebelo, J. Non-Equil.Thermodyn., 23, 351-375 (1998).

6. H.I.M. Veiga et al., Int. J. Thermophys., 22, 1159-1174 (2001).

7. L.P.N. Rebelo et al., in Liquids Under Negative Pressure, A.R. Imre, H.J. Maris, and P.R. Williams, Editors, pp. 95 - 108, NATO Science Series, Vol. 84, Kluwer Academic Publishers, Dordrecht (2002). 
8. M. Tariq, M.G. Freire, B. Saramago, J.A.P. Coutinho, J.N. Canongia Lopes, L.P.N. Rebelo, Chem. Soc. Rev., 41(2), 829 - 868 (2012).

9. M.J. Earle, J.M.S.S. Esperança, M.A. Gilea, J.N. Canongia Lopes, L.P.N. Rebelo, J.W. Magee, K.R. Seddon, J.A. Widegren, Nature, 439, 831-834 (2006).

10.M.A.A. Rocha, C.F.R.A.C. Lima, B. Schröder, L.R. Gomes, I.M. Marrucho, J.A.P. Coutinho, J.M.S.S. Esperança, J.N. Canongia Lopes, L.P.N. Rebelo, L.M.N.B.F. Santos, J. Phys Chem. B, 115, 10919-10926 (2011).

11. P. Debenedetti, Metastable Liquids, Princeton University Press (1996).

12. C.S. Santos, H.V.R. Annapureddy, N. Sanjeeva Murthy, H.K. Kashyap, E.W. Castner, Jr., and C.J. Margulis, J. Chem. Phys., 134, 064501 (2011). 